\title{
Which Provocation Test Will Be Added to Routine High-resolution Manometry Protocol in Unexplained Dysphagia?
}

\author{
Yu Kyung Cho \\ Department of Internal Medicine, The Catholic University of Korea, Seoul, Korea
}

Article: $200 \mathrm{~mL}$ rapid drink challenge during high-resolution manometry best predicts objective esophagogastric junction obstruction and correlates with symptom severity

Woodland P, Gabieta-Sonmez S, Arguero J, et al

(J Neurogastroenterol Motil 2018;24:410-414)

High-resolution manometry (HRM) is the gold standard to diagnose esophageal motility disorders. The Chicago classification of HRM findings is not complete. It sometimes does not cover all esophageal abnormalities, or explain dysphagia symptoms. ${ }^{1}$ Esophageal peristaltic defect, esophagogastric junction (EGJ) obstruction, or esophageal bolus transit abnormalities which cause dysphagia may not be detected in routine HRM protocols. ${ }^{2}$ In such cases, combining with other tests, such as timed barium esophagogram, esophageal impedance testing, or functional luminal imaging probe with HRM can be helpful to detect anatomical and functional abnormalities.

To improve the diagnostic yield of HRM without adding other esophageal motility tests, HRM provocation tests have been suggested. Provocative maneuvers are usually added on the routine HRM protocol, easy to perform in clinical practice and do not require advanced technology. Those maneuvers allows stress or load to augment abnormal esophageal response during esophageal peristalsis or transit through EGJ. ${ }^{3}$ Multiple rapid swallows (MRS), rapid drink challenge test (RDC), viscous or solid swallows, different test meals, or abdominal compression are used to provoke esophageal responses. ${ }^{3-6}$ Some tests are usually intended to measure contractile reserve, other tests are specially used to detect EGJ obstruction. Solid swallows using bread, viscous swallows, and test meals can be used to detect peristaltic disorders or EGJ obstruction. MRS is useful to evaluate contractile reserve in patients with gastroesophageal reflux disease before antireflux surgery. ${ }^{6,7}$ Abdominal compression which uses a flexible belt around the upper abdomen is useful to detect peristaltic abnormalities, especially in patients with gastroesophageal reflux disease. ${ }^{8}$

RDC test and MRS are similar provocation tests. The RDC test involves rapid drinking of $200 \mathrm{~mL}$ of liquid after the standard HRM protocol. MRS administers 5 swallows of $2-5 \mathrm{~mL}$ liquid at 2-3 second intervals. A similar physiologic responses are expected after RDC or MRS; profound deglutitive inhibition of the lower esophageal sphincter, deglutitive inhibition of esophageal body contractions, and then after rapid swallows or multiple swallows,

Received: June 16, 2018 Revised: None Accepted: June 17, 2018

(c) This is an Open Access article distributed under the terms of the Creative Commons Attribution Non-Commercial License (http://creativecommons. org/licenses/by-nc/4.0) which permits unrestricted non-commercial use, distribution, and reproduction in any medium, provided the original work is properly cited.

*Correspondence: Yu Kyung Cho, MD

Department of Internal Medicine, Seoul St. Mary's Hospital, The Catholic University of Korea, 222 Banpo-daero, Seocho-gu, Seoul 06591 , Korea

Tel: +82-2-2258-6024, Fax: +82-2-2258-2055, E-mail: ykcho@catholic.ac.kr 
the augmented contractile response follows. ${ }^{910}$ The theory of these responses requires intact neural mechanisms to regulate motility and muscular integrity to respond to the MRS or RDS stimulation. ${ }^{3}$ The absence of contractility and profound inhibition of the lower esophageal sphincter during the MRS or RDS reflects intact deglutitive inhibition. Contractile augmentation, or contractile reserve, is observed by the relative increase in distal contractile integral following MRS and defined by a ratio of post MRS contractile distal contractile integral on HRM. MRS is specially useful to assess the contractile reserve and deglutitive inhibition. ${ }^{6-8}$ As opposed to measuring contractile reserve like MRS, the best utility of RDC appears related to assessment of EGJ function. .,-11 $^{3}$

Woodland et $\mathrm{al}^{12}$ evaluated the clinical role of $200 \mathrm{~mL}$ RDC in patients with dysphagia and achalasia. RDC parameters of esophagogastric pressure gradient, integrated relaxation pressure (IRP), and RDC duration were evaluated and compared with single swallow HRM parameters or timed barium esophagogram. Mean IRP during RDC was predictable marker of EGJ outflow obstruction on time barium esophagogram, especially in untreated dysphagia patients. In patients with achalasia, mean IRP during RDC correlated with symptom scores. They concluded the parameters of RDC test predict objective EGJ obstruction and correlate with symptom severity. ${ }^{12}$

The RDC test will provide a complementary role in the evaluation of esophageal motility in dysphagia patients. However, to use it in clinical practice, it is required to define normal value and validate RDC parameters. The clinical significance of abnormal findings observed in RDC should be interpreted considering patients' symptoms in clinical practice.

\section{Financial support: None.}

Conflicts of interest: None.

\section{References}

1. Roman S, Huot L, Zerbib F, et al. High-resolution manometry improves the diagnosis of esophageal motility disorders in patients with dysphagia: a randomized multicenter study. Am J Gastroenterol 2016;111:372-380.

2. Xiao Y, Kahrilas PJ, Nicodeme F, Lin Z, Roman S, Pandolfino JE. Lack of correlation between HRM metrics and symptoms during the manometric protocol. Am J Gastroenterol 2014;109:521-526.

3. Carlson DA, Roman S. Esophageal provocation tests: are they useful to improve diagnostic yield of high resolution manometry? Neurogastroenterol Motil 2018;30:e13321.

4. Sweis R, Heinrich H, Fox M International Working Group for GI Motility and Function. Variation in esophageal physiology testing in clinical practice: results from an international survey. Neurogastroenterol Motil 2018;30:e13215.

5. Keren S, Argaman E, Golan M. Solid swallowing versus water swallowing: manometric study of dysphagia. Dig Dis Sci 1992;37:603-608.

6. Fornari F, Bravi I, Penagini R, Tack J, Sifrim D. Multiple rapid swallowing: a complementary test during standard oesophageal manometry. Neurogastroenterol Moti 2009;21:718-e41.

7. Mauro A, Savarino E, De Bortoli N, et al. Optimal number of multiple rapid swallows needed during high-resolution esophageal manometry for accurate prediction of contraction reserve. Neurogastroenterol Motil 2018;30:e13253.

8. Goldani HA, Fernandes MI, Vicente YA, Dantas RO. Lower esophageal sphincter reacts against intraabdominal pressure in children with symptoms of gastroesophageal reflux. Dig Dis Sci 2002;47:2544-2548.

9. Ang D, Hollenstein M, Misselwitz B, et al. Rapid drink challenge in high-resolution manometry: an adjunctive test for detection of esophageal motility disorders. Neurogastroenterol Motil 2017;29:e12902.

10. Marin I, Serra J. Patterns of esophageal pressure responses to a rapid drink challenge test in patients with esophageal motility disorders. Neurogastroenterol Motil 2016;28:543-553.

11. Biasutto D, Mion F, Garros A Roman S. Rapid drink challenge test during esophageal high resolution manometry in patients with esophago-gastric junction outflow obstruction. Neurogastroenterol Motil 2018;30:e13293.

12. Woodland P, Gabieta-Sonmez S, Arguero J, et al. $200 \mathrm{~mL}$ rapid drink challenge during high-resolution manometry best predicts objective esophagogastric junction obstruction and correlates with symptom severity. J Neurogastroenterol Motil 2018;24:410-414. 